\title{
TOTEM experiment at the LHC: status and program
}

\author{
Fabrizio Ferro*i \\ INFN-Genova \\ Via Dodecaneso, 33 - 16146 Genova, Italy \\ E-mail: ferro@ge.infn.it
}

The TOTEM experiment (Total Cross Section Elastic Scattering and Diffraction Dissociation at the LHC) will measure the total p-p cross section at the LHC by measuring separately the elastic and inelastic rate. The TOTEM experimental apparatus accounts for two inelastic telescopes and two Roman Pot stations per side equipped with the leading proton detectors. The detectors will be placed in the forward region of CMS: the detectors of the two experiments, operated together, provide an unprecedented rapidity coverage at a hadron collider. A common program on forward physics with different LHC running scenarios is foreseen by the two collaborations; a brief description of the part concerning soft double Pomeron exchange processes is given in this article.

DIFFRACTION 2006 - International Workshop on Diffraction in High-Energy Physics

September 5-10 2006

Adamantas, Milos island, Greece

\footnotetext{
*Speaker.

†n behalf of the TOTEM Collaboration: G. Anelli, G. Antchev, A. Arena, V. Avati, V. Berardi, U.Bottigli, M. Bozzo, E. Brucken, A. Buzzo, M. Calicchio, F. Capurro, M.G. Catanesi, S. Cerchi, M.A.Ciocci, S. Cuneo, C. Da Vià, M. Deile, E. Dimovasili, K. Eggert, F. Ferro, F. Garcia, J. Hasi, F. Haug, J. Heino, T. Hilden, P. Jarron, J. Kalliopuska, J. Kaspar, J. Kempa, A. Kok, V. Kundrat, K. Kurvinen, S. Lami, J. Lamsa, G. Latino, R. Lauhakangas, J. Lippmaa, M. Lokajicek, M. LoVetere, D. Macina, M. Macrì, M. Meucci, S. Minutoli, A. Morelli, E. Mulas, P. Musico, M. Negri, H. Niewiadomski, E. Noschis, E. Oliveri,F. Oljemark, R. Orava, M. Oriunno, K. Österberg, R.Paoletti, E. Radermacher, E. Radicioni, E. Robutti, L. Ropelewski, G. Ruggiero,A. Rummel, H. Saarikko, G.Sanguinetti, A. Santroni, S. Saramad, F. Sauli, A.Scribano, G. Sette, J. Smotlacha, W. Snoeys, F. Spinella, C. Taylor, A. Trummal, N.Turini, N. Van Remortel, S. Watts, and J. Whitmore
} 


\section{Introduction}

The TOTEM experiment at the LHC will measure[1], 2]:

- the total cross section with $\sim 1 \%$ error, by using the luminosity independent method, which requires simultaneous measurements of elastic $p p$ scattering down to the four-momentum transfer squared $-t \sim 10^{-3} \mathrm{GeV}^{2}$ and of the inelastic $p p$ interaction rate with an extended acceptance in the forward region. Present extrapolations of the world data to the LHC energy together with the existing cosmic ray data have a typical uncertainty of $\pm 15 \%$

- the elastic $p p$ scattering up to $-t \sim f e w 10 \mathrm{GeV}^{2}$

- soft diffraction

and in collaboration with CMS will

- study jets, W's and heavy flavours production in single diffratcive (SD) and double Pomeron exchange (DPE) events

- measure particle and energy flow in the forward direction

- study central exclusive production

- low-x physics.
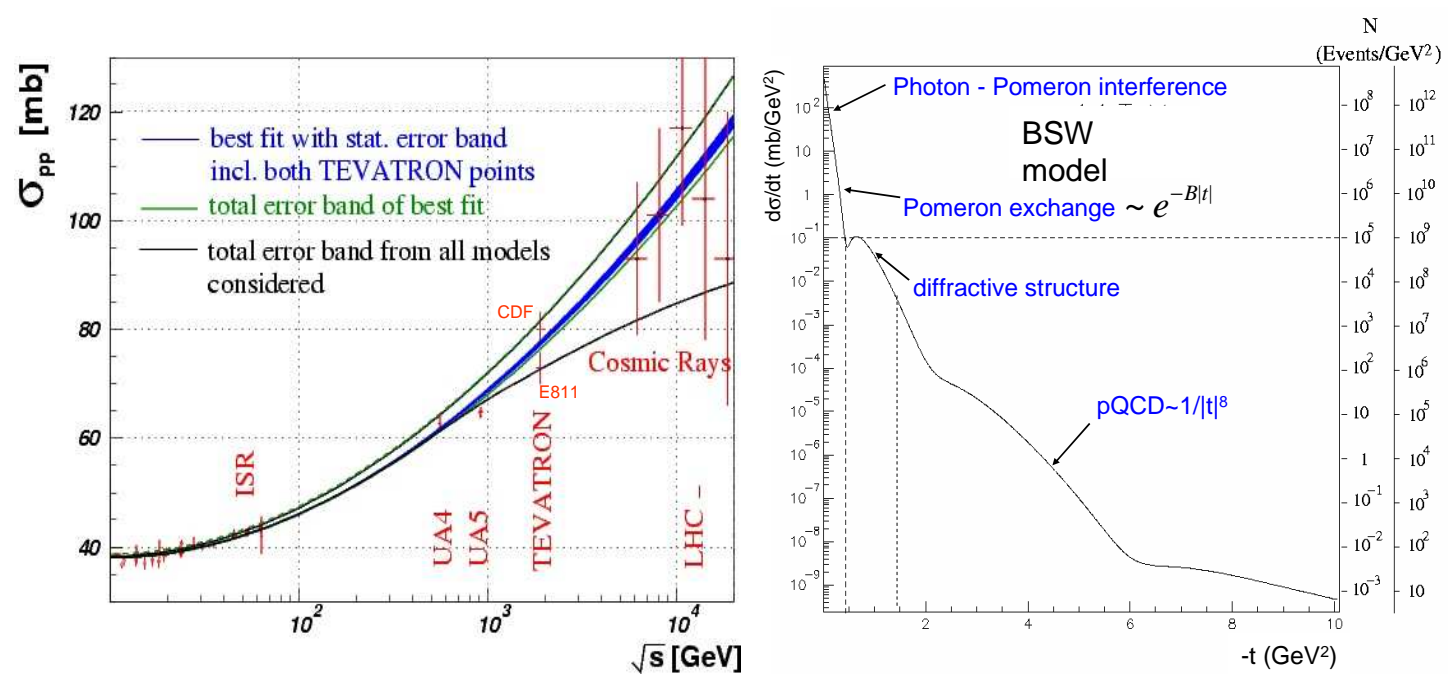

Figure 1: Left: COMPETE predictions for total $p p$ cross section [3], with PS, ISR, SPS, Tevatron and cosmic ray data. Right: elastic $p p$ cross section as predicted by the BSW model; the two columns on the right side show the number of events expected after 1 day running at $10^{28}$ and $10^{32} \mathrm{~cm}^{-2} \mathrm{~s}^{-1}$ luminosity.

The TOTEM experiment is designed to measure $\sigma_{t o t}$ with an accuracy which is sufficient to discriminate between the current model predictions for the LHC energy ranging between 100 and 
$130 \mathrm{mb}$; recent fits performed by the COMPETE collaboration[3] using all the available data, have estimated $\sigma_{t o t}=111.5 \pm 1.2_{-2.1}^{+4.1} \mathrm{mb}$ and $\rho=\frac{R e(F)}{I m(F)}=0.1361 \pm 0.0015_{ \pm 0.0025}^{ \pm 0.0058}$.

Using the optical theorem the total cross section can be written as:

$$
\sigma_{t o t}=\frac{16 \pi}{\left(1+\rho^{2}\right)} \frac{\left(d N_{e l} / d t\right)_{t=0}}{\left(N_{e l}+N_{\text {inel }}\right)}
$$

where $N_{e l}$ and $N_{\text {inel }}$ are respectively the elastic and inelastic rate.

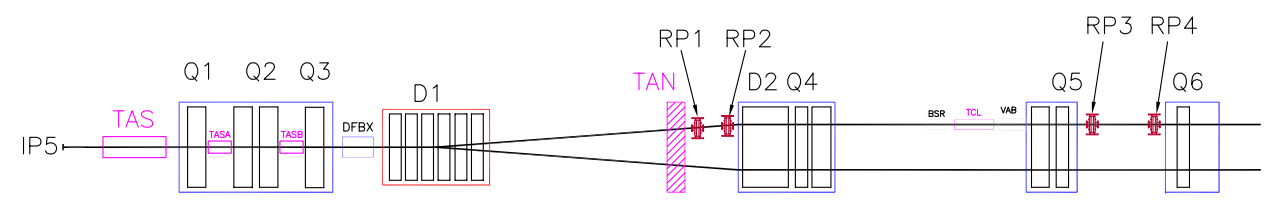

Figure 2: The LHC beam line with the Roman Pots at 147 and $220 \mathrm{~m}$.

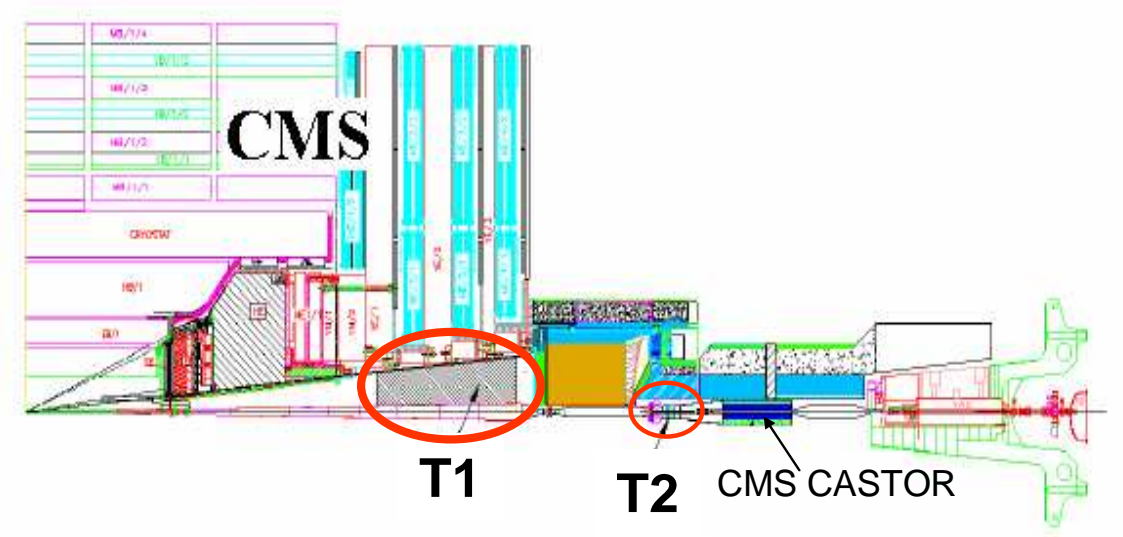

Figure 3: The TOTEM detectors $\mathrm{T} 1$ and $\mathrm{T} 2$ installed in the CMS forward region.

The precise measurement of $\sigma_{\text {tot }}$ provides also an absolute calibration of the machine luminosity:

$$
\mathscr{L}=\frac{\left(N_{e l}+N_{\text {inel }}\right)^{2}}{16 \pi\left(d N_{e l} / d t\right)_{t=0}} \cdot\left(1+\rho^{2}\right)
$$

TOTEM needs few one day runs, with the special running conditions of a high $\beta^{*}=1540 \mathrm{~m}$ (nominal TOTEM optics) and a low luminosity of $\mathscr{L} \approx 10^{28} \mathrm{~cm}^{-2} \mathrm{~s}^{-1}$. The $\beta$ value at the interaction point requires zero crossing-angle, due to the increase of the beam size (proportional to $\beta$ ), and then a reduced number of bunches which is compatible with the LHC injection scheme. The most likely configuration is 36 proton bunches with $2.5 \mu$ s spacing.

Almost half of the total cross-section at the LHC is predicted to be from coherent elastic scattering, single, double and central diffractive processes.

At $\beta^{*}=1540 \mathrm{~m}$, the TOTEM experiment efficiently detects protons with $-t>0.004 \mathrm{GeV}^{2}$, i.e. $97 \%$ of all the diffractively scattered protons, independently on their longitudinal momentum 
loss in the range of $10^{-8}<\Delta p / p<0.2$. With the TOTEM acceptance extending up to the pseudorapidities of 6.6, and with the efficient proton detection capabilities close to the LHC beams, the diffractively excited states with masses higher than $4 \mathrm{GeV} / c^{2}$ are seen by the experiment.

The precise luminosity independent measurement of the total cross section requires the measurement of $d \sigma_{e l} / d t$ down to $-t \sim 10^{-3} \mathrm{GeV}^{2}$, which corresponds to a proton scattering angle of $5 \mu \mathrm{rad}$, and the extrapolation to the optical point $(t=0)$.

The leading proton, which will be used as an event trigger, will be detected by silicon detectors placed inside movable sections of the vacuum pipe (Roman Pots), located symmetrically with respect to the interaction point (IP) (Fig. 2). In order to measure the inelastic rate, two separate forward telescopes will be installed on both sides of the CMS detector with a rapidity coverage of $3.1<|\eta|<6.6$ (Fig. 3). With these additional detectors, a fully inclusive trigger, also for single diffraction, can be provided with an expected uncertainty on the inelastic rate of the order of $1 \%$, after corrections.

\section{LHC optics}

The detection of forward protons from elastic or diffractive scattering at LHC energies requires the measurement of very small scattering angles (5-10 $\mu \mathrm{rad})$.

These particles remain close to the beam and can be detected on either side of the IP if the displacement at the detector location is large enough. The beam divergence at the IP must be small compared to the scattering angle.

To obtain these conditions, a special high- $\beta^{*}$ insertion is required. A large value $(\mathrm{O}(\mathrm{km}))$ of the $\beta$-function at the IP $\left(\beta^{*}\right)$ and a smaller beam emittance reduce the beam divergence. A large effective length $L^{e f f}$ at the detector location ensures a sizeable displacement. In fact the displacement of a scattered proton at distances from the IP $(x(s), y(s))$ is given by the proton coordinates $\left(x^{*}, y^{*}\right)$ and scattering angle $\theta_{x, y}^{*}$ at the interaction point, together with $L^{e f f}$, the magnification $v$ and the dispersion of the machine $D$ :

$$
\begin{gathered}
y(s)=v_{y}(s) \cdot y^{*}+L_{y}^{e f f} \cdot \theta_{y}^{*} \\
x(s)=v_{x}(s) \cdot x^{*}+L_{x}^{e f f} \cdot \theta_{x}^{*}+\frac{\Delta p}{p} \cdot D(s) \\
L_{x, y}^{e f f}=\sqrt{\beta_{x, y} \beta^{*}} \sin \Delta \mu(s) \quad v_{x, y}=\sqrt{\frac{\beta_{x, y}}{\beta^{*}}} \cos \Delta \mu(s)
\end{gathered}
$$

For an ideal optics, $L^{e f f}$ should be as large as possible and $v \simeq 0$, in order to reduce the dependence on the proton coordinates at the vertex. This condition of parallel-to-point focusing is achieved by requiring the betatron phase advance $\Delta \mu(s)=\pi / 2$ at the detector location, which also maximizes $L^{\text {eff }}$. The LHC optics with $\beta^{*}=1540 \mathrm{~m}$, limited by the strength of the insertion quadrupoles, provides large $L_{e f f}$ values and parallel-to-point focusing conditions in both projections at $220 \mathrm{~m}$ from the IP. This is the ideal scenario for TOTEM to measure the total cross section and to study minimum bias events and soft diffraction. 
This large- $\beta^{*}$ optics requires an injection optics different from the one which will be used at the starting runs of LHC. For this reason, an intermediate- $\beta^{*}$ optics $\left(\beta^{*}=90 \mathrm{~m}\right)$, which can use the standard LHC injection optics and can thus be operated in the first period of physics runs, has been studied. This optics provides parallel-to-point focusing only in the vertical plane and allows a measurement of $t$ only down to $-t \sim 2 \cdot 10^{-2} \mathrm{GeV}^{2}$, about one order of magnitude higher than with the nominal TOTEM optics.

\section{The experimental apparatus}

The TOTEM experiment uses precision detectors inserted in Roman Pots (movable sections of vacuum chamber) installed in the machine tunnel, at 147 and $220 \mathrm{~m}$ from the IP, to measure the elastically and diffractively scattered protons close to the beam direction. Each Roman Pot station counts for three Roman Pot units, equipped with ten planes of detectors. Two units approach the beam vertically and one horizontally, providing also an overlap among the detectors. In order to measure the elastic scattering to the smallest $|t|$ values, the detectors should be active as close to their physical edge as possible. In particular the detectors will have to be efficient up to a few tens of microns to their edge. In order to build the edgeless elastic detectors two modern technologies are being considered: planar silicon detectors with current terminating structure and planar 3D silicon detectors.

The first solution (Fig. Đ) consists in replacing the commonly used voltage terminating guard rings (usually $0.5-1 \mathrm{~mm}$ wide) with a $50 \mu \mathrm{m}$ wide structure of rings which strongly reduces the influence of the current generated at the detector edge on the active detector volume[ $₫$ ].

The second one follows a novel idea [5] which foresees that the $\mathrm{p}^{+}$and $\mathrm{n}^{+}$electrodes are processed inside the bulk of the silicon wafer, rather than being implanted on its surface as in planar devices (Fig. 丹).
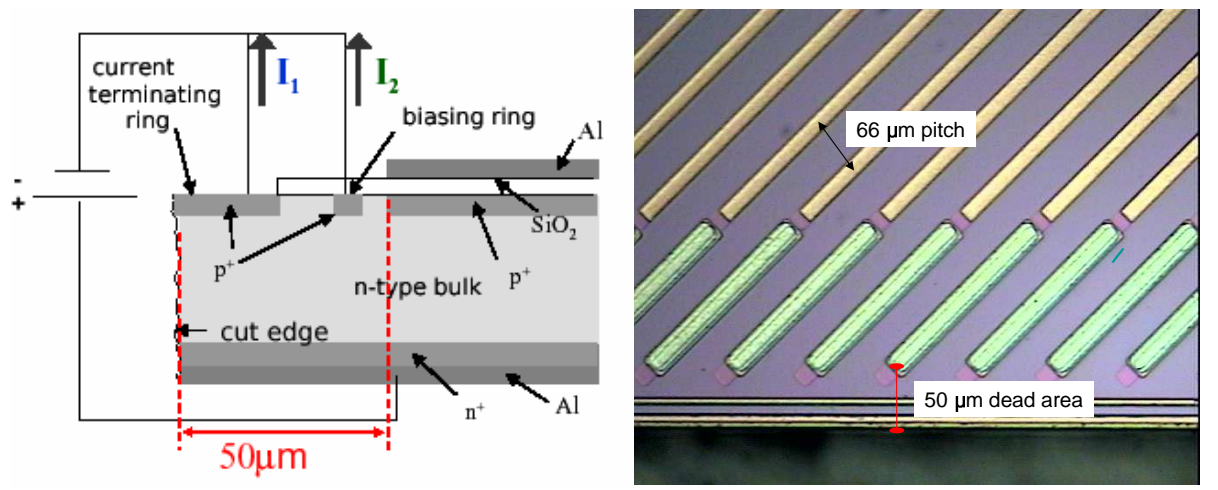

Figure 4: Left: scheme of a silicon detector with current terminating structure. Right: silicon strips in a real detector of the 2006 production.

Test-beam's performed at the CERN SPS have shown that the transition region at the edge of the 2003 prototype detectors has a width of $\sim 6 \mu \mathrm{m}$ for the $3 \mathrm{D}$ and $\sim 50 \mu \mathrm{m}$ for the cold planar. 
The telescopes for the detection of the inelastic events have a good trigger capability, provide tracking with a good angular resolution and allow the measurement of the trigger efficiency. To discriminate beam-beam from beam-gas events, the telescopes will identify the primary interaction vertex with an accuracy at the level of a $\mathrm{cm}$ by reconstructing a few tracks from each side of the interaction point; the knowledge of the full event is not needed.

The T1 telescope (Fig. 5) is made of 5 planes of 6 trapezoidal Cathode Strip Chambers (CSC) [6] and will be placed in the CMS end-caps in the rapidity range $3<|\eta|<5$ with a $2 \pi$ azimuthal coverage. T2 is made of 20 half circular sectors of GEM[7] (Gas Electron Multiplier) detectors, similar to those used for the COMPASS experiment[8]; it will be placed in the shielding behind the CMS Hadronic Forward (HF) calorimeter as a complement of the T1 telescope at larger $\eta$. With the present dimension of the vacuum pipe, the T2 telescope will cover with good efficiency the range $5.3<|\eta|<6.6$. In order to discriminate beam-beam events with respect to beam-gas ones,
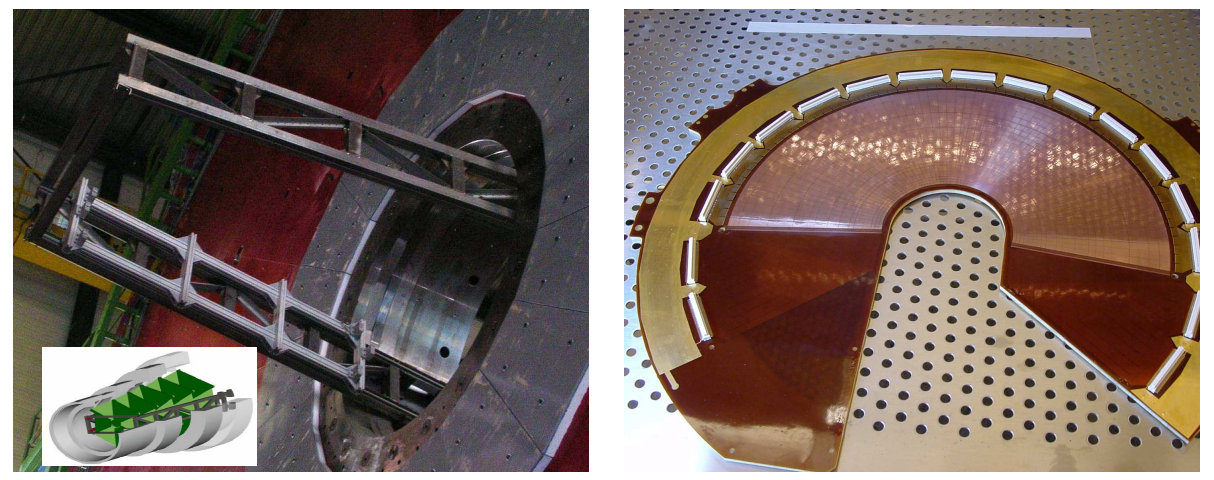

Figure 5: Left: T1 support installation inside a CMS Endcap and drawing of one arm. Right: readout board of one half-plane of the T2 triple-GEM detector.

it is necessary to achieve a good resolution in the primary vertex reconstruction. Results from the simulation of minimum bias events show that the primary vertex position in the $(\mathrm{R}, \mathrm{z})$ plane can be reconstructed with the following resolutions: $\sigma_{R}=2.9 \mathrm{~mm}$ and $\sigma_{z}=4.5 \mathrm{~cm}$.

\section{Elastic scattering}

The $x, y$ coordinates of the scattered proton are measured in the Roman Pot stations by the silicon detectors. Assuming that the edgeless silicon detectors are $100 \%$ efficient at a distance $10 \cdot \sigma_{\text {beam }}+0.5 \mathrm{~mm}$ from the beam center, one can calculate the geometrical acceptance with different beam optics in the different Roman Pot stations. In order to measure the elastic scattering at very low $t$, it is necessary to use a very high $\beta^{*}$ optics $\left(\beta^{*}=1540 \mathrm{~m}\right)$ and the Roman Pot station at $220 \mathrm{~m}$ from the IP.

As mentioned in the introduction, the measurement of the total cross section with the luminosity independent method, needs to extrapolate the rate $d N / d t$ to $t=0$. The curve of the acceptance (see Fig. (6) shows that the lowest $t$ value at which the acceptance is still $50 \%$ is $|t|=2.3 \cdot 10^{-3} \mathrm{GeV}^{2}$. Simulating a huge number of elastic events, it has been possible to estimate that the statistical uncertainty on the extrapolation can be as low as $\sim 0.1 \%$ (if one uses $10^{7}$ reconstructed elastic events, 
which corresponds to roughly 10 hours data taking at a LHC high- $\beta^{*}$ fill with a luminosity of $10^{28}$ $\mathrm{cm}^{-2} \mathrm{~s}^{-1}$ ).

The extrapolation accuracy due to experimental effects is much more important than that due to the statistics. The main sources of systematic uncertainties are the beam divergence, the beam energy and the beam position offset.

The effects of a non-exponential behaviour of $d \sigma / d t$ have to be taken into account when extrapolating to the optical point: even at low $|t|$ there may be important deviations of the hadronic cross section from the exponential behaviour. The influence of the Coulomb scattering becomes significant for $|t|<0.01 \mathrm{GeV}^{2}$. The systematic error on the extrapolation to $t=0$ due to the theoretical uncertainties of the functional form has been estimated to be less than $0.1 \%$. For the intermediate$\beta^{*}$ optics the error grows up to $0.5 \%$, but it is partially compensated by a smaller contribution from the other systematic effects.

The final overall uncertainty on the extrapolated elastic rate has been estimated to be $\sim 0.5 \%$.
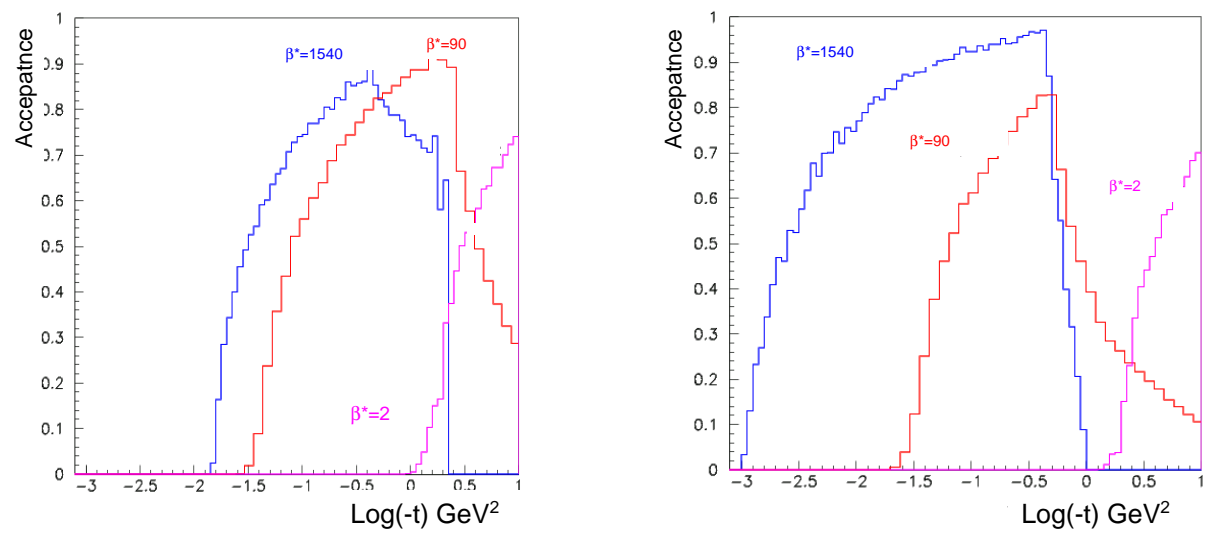

Figure 6: Geometrical acceptance of the detectors at 147 (left) and $220 \mathrm{~m}$ (right) with three different beam optics.

\section{Inelastic scattering and total cross section}

The measurement of the inelastic rate requires a trigger as inclusive as possible and a clear discrimination of beam-beam events from background interactions. With a double arm trigger more than $99 \%$ of non-diffractive events can be detected, while with a single arm one can detect the events escaping the double arm trigger and most of the diffractive interactions with all visible tracks in one arm only. The main background is due to beam-gas events, which can be rejected with an accurate primary vertex reconstruction. Simulation studies show that only $\sim 3 \%$ of the beamgas events are misidentified as beam-beam, corresponding to a rate well below $1 \mathrm{~Hz}$. Moreover, the detection of the surviving proton, with more than $90 \%$ efficiency, can be a powerful tool in the discrimination between beam-gas and single diffractive events.

The studies show that single and double diffractive events are responsible for the major loss in the inelastic rate. With a single arm trigger, a fraction of these events, corresponding to $\sim 2.8 \mathrm{mb}$, 
escapes detection. The lost events are mainly those with a very low mass (below $\sim 10 \mathrm{GeV} / c^{2}$ ), since all the particles are generated at pseudo-rapidities above 7 and escape therefore the detection.

To obtain the total inelastic rate, the fraction of events lost because of the incomplete angular coverage can be estimated by extrapolation. In the case of single diffraction, the reconstructed $1 / M^{2}$ distribution has been linearly fitted for $M>10 \mathrm{GeV} / c^{2}$ and the extrapolation to low masses has then been compared with the Monte Carlo. For single diffraction, the extrapolated number of events differs from the Monte Carlo by $4 \%$, corresponding to a $0.6 \mathrm{mb}$ uncertainty on the total cross section. The same estimation for the double diffraction and double Pomeron exchange gives $0.1 \mathrm{mb}$ and $0.2 \mathrm{mb}$ of uncertainty respectively.

The combination of the elastic and inelastic scattering uncertainties, after the extrapolations, gives a final error on the total cross section of $\sim 1 \%$.

\section{Diffraction}

The CMS and TOTEM detectors are installed at the same interaction point and, when operated together, form the experiment with the largest rapidity acceptance ever built at a hadron collider. The resulting coverage provides an ideal experimental apparatus to study diffraction and, more generally, many aspects of the forward physics in proton-proton collisions. The two collaborations have been preparing an extensive common physics program which describes the CMS/TOTEM perspective about soft and hard diffraction (mainly in single diffractive and double Pomeron exchange - DPE - events), $\gamma-\gamma$ and $\gamma$-p physics, forward production of Drell-Yan pairs, connections between forward and astro-particle physics.

Different LHC running scenarios can be used to investigate different aspects of the physics program; a small number of runs with a high quality beam and low $\left(10^{28}-10^{30} \mathrm{~cm}^{-2} \mathrm{~s}^{-1}\right)$ luminosity is ideal to study minimum bias and soft diffraction, on the other hand a higher luminosity and a much longer data taking is necessary to study processes with low cross sections, like jet-jet events production or, even more challenging, the Higgs production in double Pomeron exchange events.

\subsection{Soft DPE}

In DPE events, the two protons emerge from the scattering and a central system of mass $M=\sqrt{\xi_{1} \xi_{2} s}$ is created. For the LHC energy the kinematical limit for the central system mass is roughly $1.4 \mathrm{TeV}$. The cross section of such processes is expected to decrease sharply with the mass (approximately $d \sigma / d M^{2} \propto 1 / M^{2}$ ), but nevertheless a huge amount of data is expected to be taken in a few days even at rather low luminosities (e.g. at $\mathscr{L}=10^{29} \mathrm{~cm}^{-2} \mathrm{~s}^{-1}, \sim 10000$ events with $\mathrm{M}=50 \mathrm{GeV} / c^{2}$ are expected in one day running). Moreover, due to the particular topology, DPE events can be triggered with the almost background free condition of requiring the detection of a proton per each side and at least a track in the T1/T2 telescopes.

The protons emerging from diffractive scattering at LHC are emitted at very small angles (10-150 $\mu \mathrm{rad})$; they undergo a momentum loss $(\xi=\Delta \mathrm{p} / \mathrm{p})$ of $10^{-7} \div 0.1$ and, if the displacement from the nominal beam line is large enough, they can be detected in the leading proton detectors. In order to reconstruct the path of the proton through the magnetic elements, the optical functions (see eqs. 2.1, 2.2) need to be known; they depend not only on the position along the beam line, but 
also on the proton parameters at the interaction point. For DPE events, the geometric acceptance of the Roman Pot at $220 \mathrm{~m}$ starts at $-\mathrm{t}$ values of $3 \cdot 10^{-2} \mathrm{GeV}^{2}$ for $\beta^{*}=90 \mathrm{~m}$, at $1 \cdot 10^{-3} \mathrm{GeV}^{2}$ for $\beta^{*}=1540 \mathrm{~m}$ and, in both cases, it is almost independent on the proton momentum loss $\xi$ (Fig. ฤ). The overall proton accepatance in DPE events is almost $90 \%$ for the high $\beta^{*}$ optics and about $50 \%$ for the intermediate one.
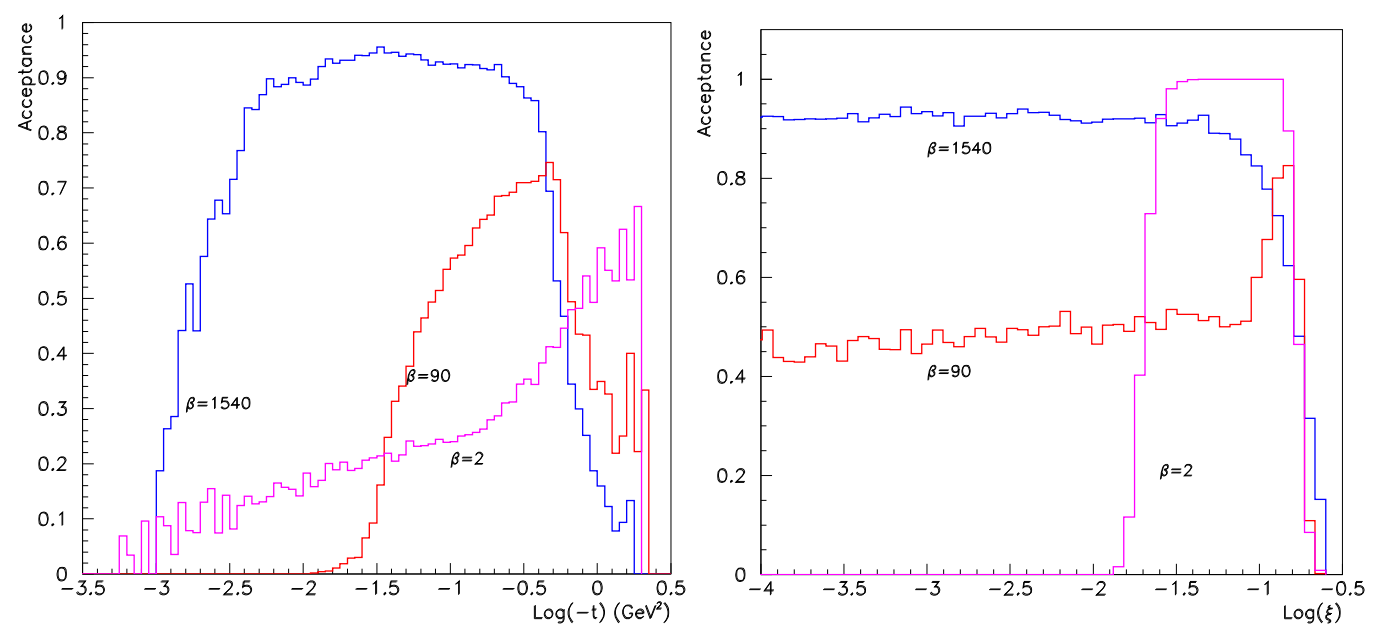

Figure 7: Proton acceptance at different $\beta^{*}$ for the Roman Pots at $220 \mathrm{~m}$ (the schematic contour plot refer to a minimum acceptance of $30 \%$ ) vs $t$ (left) and $\xi$ (right).

Since the nominal TOTEM optics is optimized to have a high acceptance in $\mathrm{t}$ with poor requirements on the $\xi$ resolution, the mass resolution ranges from $30 \mathrm{GeV} / \mathrm{c}^{2}$ for symmetric events (where protons undergo the same momentum loss) to $250 \mathrm{GeV} / c^{2}$ for very asymmetric. A better resolution (from 15 to $60 \mathrm{GeV} / c^{2}$ ) is on the contrary expected for the intermediate $\beta^{*}$ optics.

In order to improve the mass resolution, indirect measurements of the proton momentum loss can be done using the wide rapidity range covered by CMS and TOTEM detectors. One method, used in the past by CDF[9] and D0[10] at the Tevatron, utilizes the energy measurements from the calorimeters, correlated with the proton momentum loss by the relation $\xi^{ \pm} \sqrt{s}=\sum_{i} E_{T}^{i} e^{ \pm \eta_{i}}$, where $E_{T}^{i}$ is the trasverse energy of the i-th particle at pseudorapidity $\eta_{i}$. With this method a resolution on $\xi$ of roughly $40 \%$ is expected.

Another way to measure $\xi$ is using the measurement of the rapidity gap width by means of trackers and calorimeters. The simulated data in Fig. 8 show the linear relation between $\xi$ and the gap. With this method a resolution of $\sim 80 \%$ can be achieved. The possibility of doing indirect measurements of $\xi$ can be of great importance in the region of very low $\xi\left(\xi<10^{-3}\right)$, where the resolution of the direct measurement can easily exceed $100 \%$.

\section{Conclusion}

The main aspects of the TOTEM scientific program are the measurement of the total p-p cross section and of the elastic scattering up to $-t=8 \mathrm{GeV}^{2}$. For this part of its program TOTEM will use dedicated short runs of the LHC with high $\beta^{*}$ optics and low luminosity. 

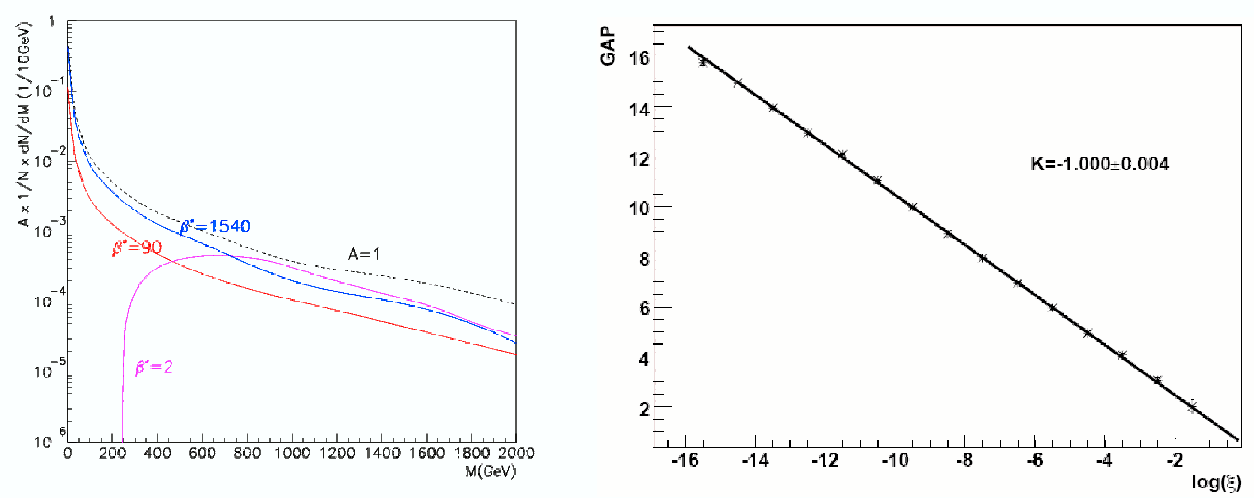

Figure 8: Left: DPE differential cross section with and without proton acceptance for different optics. Right: measured gap vs proton $\xi$ with a linear fi t.

The study of the physics performance has shown that an accuracy of $\sim 1 \%$ on the value of the total cross section is achieveble with the experimental apparatus, which is being built and which will be installed in the IP5 cavern in time for the starting of the LHC operations.

CMS and TOTEM collaborate for the realization of an extesive physics program on diffraction and, more generally, on many aspects of the forward physics.

\section{References}

[1] The TOTEM collaboration, Technical Design Report, CERN-LHCC-2004-02.

[2] The TOTEM collaboration, Technical Design Report - Addendum, CERN-LHCC-2004-20.

[3] J. R. Cudell et al., Phys. Rev. Lett. 89, (2002) 201801.

[4] G. Ruggiero et al., Planar edgeless silicon detectors for the TOTEM experiment, Proc. IEEE, Vol. 52 (2005).

[5] S. I. Parker, C. J. Kenney and J. Segal, NIM A 395 (1997) 328.

[6] CMS MUON Technical Design Report, CERN/LHCC 97-32, CMS TDR 3, 15 December 1997.

[7] F. Sauli, GEM: a new concept for electron amplification in gas detectors, NIM A 386, (1997) 531.

[8] C. Altunbas et al., Construction, test and commissioning of the triple-gem tracking detector for Compass, NIM A 490 (2002) 177.

[9] D. Acosta et al., CDF Collaboration, Phys. Rev. Lett. 93 (2004) 141601.

[10] V. M. Abazov et al., D0 Collaboration, Hard single diffraction in $p-{ }^{-p}$ collisions at $\sqrt{s}=630 \mathrm{GeV}$ and $1800 \mathrm{GeV}$, Phys. Lett. B531 (2002) 52. 